\title{
Correction to: Magnetic Nanoparticles and Alternating Magnetic Field for Cancer Therapy
}

Harutaka Mekaru, Yuko Ichiyanagi, and Fuyuhiko Tamanoi

\section{Correction to:}

Chapter 7 in: D. O. Wang, D. Packwood (eds.), Cell-Inspired Materials and Engineering, Fundamental Biomedical Technologies, https://doi.org/10.1007/978-3-030-55924-3_7

The name of one of the author of this chapter should be: Yuko Ichiyanagi which was mistakenly printed as Yuko Ichivanagi. The error has been corrected in this chapter. 